\title{
Ambientes colaborativos virtuales para el aprendizaje individual
}

Virtual collaborative environments for individual learning

\author{
Volumen 17, Número 2 \\ Mayo-Agosto \\ pp. 1-29
}

Este número se publica el $1^{\circ}$ de mayo de 2017

DOI: http://dx.doi.org/10.15517/aie.v17i1.28092

Denis González Herrera

Revista indizada en REDALYC, SCIELO

Revista distribuida en las bases de datos:

LATINDEX, DOAJ, $\underline{\text { REDIB }}$ IRESIE, $\underline{\text { CLASE}}$, DIALNET, SHERPA/ROMEO, QUALIS-CAPES, MIAR

Revista registrada en los directorios:

ULRICH'S, $\underline{\text { REDIE}}, \underline{\text { RINACE}}, \underline{\text { OEI }}$ MAESTROTECA, PREAL, $\underline{\text { CLACSO }}$ 


\title{
Ambientes colaborativos virtuales para el aprendizaje individual
}

\author{
Virtual collaborative environments for individual learning
}

\section{Denis González Herrera ${ }^{1}$}

\begin{abstract}
Resumen: El presente artículo muestra los resultados de una investigación sobre ambientes colaborativos virtuales, en la que participó un grupo de 15 estudiantes de la Universidad de Costa Rica. El objetivo de esta fue innovar en la formulación de estrategias didácticas que ayuden a involucrar al estudiantado-profesorado, como actores en los procesos de formación. Lo anterior se logró mediante el empoderamiento de tecnologías de la información y comunicación (TIC) y, en especial, el uso de dispositivos móviles. La investigación se realizó en el 2014, desde una aproximación mixta que sistematiza el proceso, experiencias y aprendizajes recolectados. Los datos se recopilaron por medio de un instrumento autoadministrado, en un único momento, además, las observaciones de los participantes fueron registradas durante todo el ciclo. La investigación ayudó a identificar herramientas tecnológicas que permiten innovar en la creación de ambientes colaborativos; además, se logró evidenciar que estos aumentan y mejoran la experiencia del proceso de aprendizaje. De igual forma, se demuestra que pueden ser creados con herramientas de software libre y accesible; y que se requieren habilidades mínimas de computación para personalizarlo, según las necesidades educativas. Por último, se expone una propuesta de un modelo de cinco etapas que permite que otros docentes puedan replicar este tipo de ambientes; el cual fue guía en la creación de una aplicación para celulares completamente funcional, que puede ser objeto de estudio en una futura investigación.
\end{abstract}

Palabras clave: Ambiente colaborativo, Entorno virtual, aprendizaje, TIC, dispositivos móviles, M-learning, Educación superior.

\begin{abstract}
This article presents the research results about virtual collaborative environments, in which a group of 15 students from the University of Costa Rica participated. The objective of the research was to innovate in the formulation of methodological and pedagogical techniques that help to involve the student-instructor, as actors in the training processes. This was achieved through the empowerment of information and communication technologies (ICT) and especially using mobile devices. The research was carried out in 2014, from a mixed approach that systematizes the process, experiences and lessons learned. The data were obtained by a selfadministered instrument, supplied in a single moment; moreover, the observations of the participants were recorded during the whole cycle. The research helped to identify technological tools that allow innovating in the creation of collaborative environments; in addition, it was demonstrated that these increase and improve the experience of the learning process. It also demonstrates that the collaborative environments can be created with free and accessible software tools; and that minimal computer skills are required to customize it according to educational needs. Finally, a proposal is presented for a five-stage model that allows other teachers to replicate this type of environment; which was a guide in the creation of a fully functional mobile application that can be studied in future research.
\end{abstract}

Keywords: Collaborative environment, virtual environment, learning, ICT, mobile devices, M-learning, superior education.

1 Docente del Departamento de Ciencias Naturales, Sede
Occidente, Universidad de Costa Rica.

Dirección electrónica: dennis.gonzalezherrera@ucr.ac.cr

Artículo recibido: 16 de junio, 2016

Enviado a corrección: 25 de octubre, 2016

Aprobado: 13 de febrero, 2017 


\section{Introducción}

En la era contemporánea, la sociedad está inmersa en un mundo dependiente de la utilización de los dispositivos tecnológicos como: dispositivos móviles, redes sociales y tecnologías de comunicación digital, entre otros. De igual forma, las personas son participantes directos como agentes pasivos, ya que realizan un alto consumo de información digital a través de estos medios sin llegar a crear o redefinir las TIC, según sus preferencias (no se apropian de la tecnología). Los entornos universitarios no están libres del impacto de estos cambios en nuestra era digital; docentes y estudiantes pueden verse beneficiados con la incorporación de artefactos digitales que faciliten y mejoren los ambientes de aprendizaje.

El proceso de enseñanza-aprendizaje mediado por las TIC da la posibilidad de adaptar las necesidades y características de los usuarios en su formación y preferencias (Ferro, Martínez y Otero, 2009). "Es habitual hablar de este tiempo de cambios, propiciado por los avances de las tecnologías de la información y la comunicación, como del inicio de una nueva era, a la que suele llamarse sociedad de la información" (Ibáñez, 2004, p. 2). Con el libre acceso a la tecnología es posible diseñar y crear entornos virtuales que se adapten y sean personalizados a las necesidades educativas; que faciliten la colaboración de ideas y maximicen el tiempo disponible en la elaboración, consumo y selección de contenidos, acorde con sus necesidades.

Salinas (2005, p. 1) indica que "Los ambientes educativos, tal como los conocemos, han comenzado a transformarse notoriamente en la actualidad para adaptarse a la sociedad de la información". Sin embargo, la tecnología por sí sola no cambia el modelo educativo, las prácticas didácticas ni los paradigmas presentes; no es capaz de resolver los problemas y retos inmersos en los escenarios universitarios. Marino (2013, p. 13) indica que las instituciones educativas utilizan el aprendizaje del ciberespacio como menciona él, para continuar realizando las mismas prácticas de aprendizaje sin atender nuevas necesidades. Con sus palabras se resume: “(...) se utilizan nuevas tecnologías para seguir reproduciendo viejas concepciones pedagógicas". Se nos ha olvidado que el sujeto del aprendizaje es un ser creador por antonomasia.

Según Ibáñez (2004), se debe hacer énfasis en la docencia, en las estrategias didácticas del profesorado, en la comunicación y distribución de los sistemas de aprendizaje (procesos de innovación docente apoyados en las TIC) y no concentrar esfuerzos en la disponibilidad y las potencialidades tecnológicas. La adquisición de tecnología, el equipamiento, el simple uso de la tecnología no cambian el panorama educativo. Se hace necesario instruir al profesorado, 
primeramente en el potencial tecnológico existente, en las habilidades, competencias necesarias y en las pericias que le ayuden a encontrar las formas de interiorizar los conocimientos requeridos de la cultura digital. Cualidades que permitan hacer frente a los retos y necesidades que deben superar las nuevas generaciones. "Las TIC contribuyen al vertiginoso cambio que exige nuevas destrezas y cambios en los objetivos, pueden contribuir a su logro y dominio" (Salinas, 2005, p. 2).

De igual forma, el estudiantado cuenta con nuevas destrezas en materia de redes digitales, manejo de dispositivos móviles y un alto consumo de todo tipo de información electrónica; que, por un lado, consume el tiempo disponible del estudiantado para labores universitarias. Este hecho puede ser un obstáculo si se quiere invertir en aprendizajes de calidad; pero, por otro lado, todas estas pericias y experiencias pueden ser reenfocadas en la búsqueda de la excelencia académica. De esta manera, los actores, docente-estudiante, se acercan a la tecnología como agentes activos y partícipes de su propio destino. Tal como mencionan Moreno y Molano (2012, p. 6) "la sociedad del conocimiento es una oportunidad para que quienes estén fuera de ella, ingresen y se conviertan en actores del nuevo escenario global".

Tanto el profesorado como el estudiantado pueden empoderarse de las tecnologías digitales, para moldear el mundo a su alrededor, mejorar los modelos y paradigmas de enseñanza-aprendizaje actuales; y también crear nuevos enfoques que consideren la experticia de las generaciones modernas y guíen al personal docente en procesos orientadores que respondan a las necesidades actuales.

Por medio del trabajo de sistematización, se aporta el proceso y el esfuerzo empleado en materia de ambientes virtuales con aspecto colaborativo que incentivan al estudiantado en su aprendizaje personal. Se describe su papel generador e interactivo como participe en el diseño de aplicaciones que lo ayuden de forma individual en la apropiación creadora de los contenidos programáticos. Se pretende incentivar a la comunidad docente en la innovación de prácticas metodológicas y pedagógicas que faciliten la apropiación de la tecnología en el proceso enseñanza-aprendizaje; al mismo tiempo, se formulan nuevas alternativas en el uso de herramientas TIC y se exponen ideas transformadoras que permitan trascender en la labor diaria. De igual forma, menciona Salinas (2005, p. 3): "Describir escenarios de aprendizaje propiciados por las TIC nos ayudará en el diseño y creación de ambientes de aprendizaje adecuados a las nuevas coordenadas espaciotemporales, a los nuevos objetivos educativos $(\ldots)$. 
Durante el ejercicio académico fue posible la creación de una propuesta constituida de cinco momentos principales llamados: motivación, modelado de ideas, integración de conocimiento, ejecución y reconstrucción; la cual fue ideada como una espiral para permitir la continuación del trabajo. Lo anteriormente expuesto por cuanto se considera que el proceso de enseñanza-aprendizaje no tiene un final propiamente diseñado; y la obtención de conocimiento puede ser la base para alcanzar el nuevo. Desde esta perspectiva epistemológica, no interesa la reproducción del conocimiento, sino la creación del conocimiento.

El trabajo en las etapas señaladas anteriormente permitieron modelar y crear un ambiente digital, facilitador. Esto fue posible al utilizar las especificaciones y aportes recogidos en cada uso e interacción de los participantes con el entorno virtual; demostrando la accesibilidad de la tecnología. Por último, se aportan los resultados e impresiones obtenidas de la observación sobre la puesta en funcionamiento del entorno virtual. Se consideró la exploración de ideas y la recopilación de experiencias del estudiantado, las cuales fueron conseguidas por medio de un cuestionario con preguntas abiertas y preguntas cerradas, las respuestas demuestran la aprobación de las TIC dentro y fuera del aula, evidencian el apoyo de la metodología y las TIC a la labor docente, e invitan a la formulación de cambios en los modelos educativos actuales.

\section{Marco teórico referencial}

A continuación, se presentan las definiciones sobre conceptos, herramientas y temas importantes para una adecuada comprensión de la investigación. Asimismo, se describe su relación, importancia y su influencia sobre el actual estudio.

\subsection{Tecnologías de información y comunicación (TIC)}

La terminología que envuelve a las TIC es amplia y es utilizada para referenciar diferentes facetas o características; y puede cambiar dependiendo del tipo de institución, persona u organismo. Según Cobo (2011), es posible categorizarlas en herramientas, usos e impacto; expone que son las tecnologías relacionadas principalmente con las áreas de: hardware, software, electrónica, informática y telecomunicaciones. Estos elementos permiten realizar diversas acciones como: el acceso, almacenamiento, procesamiento y distribución de datos entre distintos medios y formas diferentes. Las TIC provocan un impacto directo sobre 
la sociedad moderna, la importancia del conocimiento y los retos alrededor de las nuevas competencias requeridas para su correcto manejo.

Para la presente investigación, es pertinente plantear la postura conceptual acotada en el papel de las instituciones educativas, el posicionamiento del personal académico-estudiantil y el impacto sobre la sociedad del conocimiento. De esta forma, TIC se entiende como el hardware y software que brinda la posibilidad de interactuar con datos (editar, crear, almacenar o transmitir), mediante distintos sistemas de información que integran medios de informática, telecomunicaciones o redes. Además, permite la comunicación interpersonal o multidireccional y posibilita un impacto sustantivo al conocimiento (Cobo, 2011).

Uno de los mayores retos de las tecnologías es el generado por su rápido crecimiento y difusión, el cual obliga a modernizar a las instituciones educativas; las cuales deben asimilar e integrar de forma ágil y oportuna estos cambios a sus procesos de enseñanza-aprendizaje. Deben conceptualizar nuevos actores: docentes y estudiantes con nuevas habilidades, destrezas y competencias básicas sobre los avances tecnológicos. Las TIC brindan el mecanismo para implementar puentes para la comunicación y para la interacción dentro y fuera del espacio físico educativo, y de esta manera poner a disposición de la educación, las habilidades tecnologías de las personas docente- estudiante.

\subsection{Aprendizaje móvil o M-learning}

En el marco de las TIC se posicionan las tecnologías para el aprendizaje mediante dispositivos móviles, como teléfonos inteligentes o tabletas electrónicas. Son un instrumento que, por su amplia difusión, acceso y utilización en la sociedad moderna, facilitan la comunicación y la omnipresencia de la información, siendo un mecanismo ideal como herramienta facilitadora y de mediación en los procesos de aprendizaje. Según Conde (2007), es una oportunidad para aprovechar las facilidades de las tecnologías portátiles; como dar sostén y ser el camino dirigido a un proceso de aprendizaje ubicuo en la sociedad.

Desde esta misma línea, el aprendizaje móvil presenta virtudes para los procesos educativos al enriquecer, estimular y dar flexibilidad a los usuarios; posibilitando el desarrollo académico desde lugares cotidianos y facilita la incorporación en el escenario de aprendizaje, a las variables intimas de los participantes: espontaneidad, informalidad, personalidad, portabilidad, comodidad y disponibilidad. Son estas cualidades las que hacen necesario el desarrollo de herramientas y mecanismos de dispositivos móviles como mediación en los ambientes de enseñanza-aprendizaje. 


\subsection{Appinventor}

Es un entorno basado en las TIC que puede ser empleado de manera libre para la creación de aplicaciones que trabajen sobre dispositivos móviles que cuenten con el sistema operativo android. Esta plataforma fue creada por la empresa Google y actualmente es liderada por el Instituto Tecnológico de Massachusetts, con la modalidad de software libre. Gracias a esta, se crean aplicaciones para dispositivos móviles y puede ser utilizada por cualquier usuario que cuente con una dirección de correo electrónico perteneciente a la empresa Google. Autores como Bianchi, Lombardero, Fernández, y Barrios (2014) indican que es una herramienta motivadora, sencilla de utilizar para principiantes o expertos; puede ser empleada con facilidad en la creación de prototipos rápidos y funcionales sin perder tiempo en actividades producidas por errores de programación, de sintaxis, compilación o ejecución.

El Appinventor muestra características como: la rapidez de desarrollo, reduce el tiempo requerido en la implementación de nuevas aplicaciones; cuenta con un entorno, gráfico completamente visual y amigable, basado en la idea de "tomar y arrastras", objetos de programación como si fueran piezas que deben ser unidas. Estas características permiten que las personas con pocos o ningún conocimiento en lenguajes de computadora puedan diseñar y crear aplicaciones empleadas en la labor diaria (Ascheri, Testa, Pizarro, Camiletti, y Diaz, 2014).

La plataforma se encuentra completamente ambientada en el concepto de Cloud Computing o computación en la nube; esta característica la coloca al alcance de los usuarios que cuenten con conexión a internet. Asimismo, permite guardar los proyectos realizados, los cuales pueden ser accedidos desde cualquier lugar a través de distintos medios electrónicos.

Recapitulando, la plataforma de Appinventor es accesible para cualquier persona que posea una cuenta de correo electrónico de Google; con o sin conocimientos previos en programación, facilita la creación de aplicaciones para dispositivos móviles con el sistema operativo Android; y da la posibilidad para que las personas sean creadoras de este tipo de aplicaciones móviles, y no únicamente simples usuarios. De igual forma, es aclarado por Observatorio de la formación en Red SCOPEO, 2011: "herramienta de autor a resaltar, pensada y pensando en animar a los "no programadores" a ser no solo consumidores de aplicaciones, sino también creadores" (p. 154). 


\subsection{Cloud computing, Google AppsEngine}

Puede ser conceptualizado de distintas maneras según el tipo de servicio, proveedor, cliente o mercado. Bertolín (2010, p. 42) afirma: "Un estilo de computación donde las capacidades relacionadas con las TIC se proporcionan de forma masivamente escalable que se facilita como un servicio utilizando las tecnologías de Internet dando soporte a múltiples clientes externos".

Para el mismo autor este concepto es definido como:

Un modelo para permitir un acceso a red conveniente y bajo demanda a un conjunto compartido de recursos de computación configurables (redes, servidores, almacenamientos, aplicaciones y servicios) que pueden proporcionarse rápidamente con un mínimo de esfuerzo de gestión o de interacción del proveedor del servicio (definición del NIST). Este modelo o paradigma de nube promueve la disponibilidad. (Bertolín, 2010, p. 42)

Sobre la misma línea se define como un paradigma basado en las herramientas computacionales que permiten el intercambio de información multidireccional entre múltiples usuarios, mediante una amplia clase de dispositivos electrónicos, de forma rápida y accesible en un entorno de ubicuidad.

Algunos de los principales proveedores de este tipo de servicios son: Google AppsEngine, Amazon Web Services, Microsoft Windows-Azure. Esteve (2009) señala como ejemplo de servicios ofrecidos por AppsEngine a: Google Docs, Gmail, Google Calendar o Google drive; los cuales dan al usuario acceso a documentos, correo electrónico, agenda personal y espacio de almacenamiento respectivamente a partir de cualquier dispositivo conectado a internet. También brinda la posibilidad de compartir y realizar actividades de colaboración con su información y con la de otros usuarios. Según el ranking de las cien herramientas tecnológicas más utilizadas para el aprendizaje en el 2016, Centre for Learning and Performance Technologies (C4LPT) destaca herramientas colaborativas como: Google Docs y Drive en la quinta posición y Google Apps en el puesto cuarenta.

\subsection{Entorno virtuales de aprendizaje}

Los conceptos antes mencionados representan los recursos básicos sobre los cuales es posible la implementación de espacios académicos adicionales y complementarios a los cursos físicos tradicionales. Nos referimos a los entornos virtuales, los cuales son una 
representación o copia de un escenario académico real, donde se expresan los papeles de todos los participantes, las actividades, las formas de interacción y participación entre ellos; pero agregando nuevas cualidades brindadas por la tecnología. También es posible conceptualizarlo desde un punto de vista técnico, al describir las TIC que están envueltas en la creación, utilización y vinculación de la información; y que facilitan el proceso de enseñanzaaprendizaje.

Algunas concepciones que respaldan estas afirmaciones son: "Espacio en un ordenador gestionado por un programa de trabajo colaborativo que permite el acceso restringido a los miembros que hayan sido invitados a dicho entorno a través de internet" (Alcina, 2002, p.172). En la misma línea, Catasús, Romeu y Pérez-Mateo (2007) indican que los entornos virtuales ofrecen oportunidades ineludibles para el desarrollo de proyectos basados en metodologías de enseñanza-aprendizaje y trabajo cooperativo.

"Como representación de la institución educativa y de todas las actividades semióticas que en ella acontecen y como dominio semiótico que articula y da lugar a distintas prácticas y textos multimodales en diversos tipos de interacciones" (Imperatore, 2009, p.111). Con base en lo indicado anteriormente, además de entender el entorno virtual como un elemento tecnológico, hay que agregar que representa a la entidad educativa en todas sus formas, métodos, prácticas y actividades; también en ella se engloba la manera como es concebida por quienes enseñan y quiénes aprenden, las formas cómo generan y cómo interiorizan el conocimiento, limitando al mismo tiempo las fronteras de lo permitido y lo no permitido.

\subsection{Ambientes colaborativos}

Un pilar importante en los procesos y en los espacios educativos es la colaboración, la cual se beneficia de las TIC. De acuerdo con Calzadilla (2002) en su artículo titulado, Entornos colaborativos y tecnologías de la información y la comunicación: "los individuos que intervienen en un proceso de aprendizaje, se afectan mutuamente, intercambian proyectos y expectativas y replantean un proyecto mutuo, que los conduzca al logro mutuo de un nuevo nivel de conocimiento y satisfacción".

En este tipo de aprendizaje, cada participante decide una postura sobre su ritmo de trabajo, sus potencialidades y sobre su actividad personal; al mismo tiempo, debe aportar lo mejor de sí mismo al trabajo grupal, con el objetivo de lograr metas que no podrían alcanzar solos o requiere un esfuerzo mucho mayor. Este tipo de interrelaciones fortalece el crecimiento 
individual, aumenta la productividad y mejoran las relaciones interpersonales. (Calzadilla, 2002)

La experiencia en este tipo de entornos propicia la igualdad, motivación y la bidireccionalidad de información, permite la retroalimentación y estimula al estudiantado a la búsqueda de un aprendizaje personal e individual. Consecuentemente, fomenta un fuerte sentimiento de unión, pertenencia y responsabilidad en alcanzar las metas comunes; es importante la participación de un autor mediador que guíe el proceso de aprendizaje, brinde seguridad y ayude a lograr la apropiación del conocimiento y lo transfiera al medio. Para Carrió (2007, p. 2): "el aprendizaje colaborativo es una propuesta de enseñanza-aprendizaje basada en los conceptos de cooperación, trabajo en equipo, comunicación y responsabilidad". Este es un espacio donde se aprende a compartir conocimiento, incrementa la autoestima, seguridad y motivación mediante la colaboración de responsabilidades, estimula la toma de decisiones grupales, permitiendo al estudiantado conocer su ritmo de aprendizaje, con la ayuda de un mediador que gestione y corrija las acciones.

\subsection{Aprendizaje individual}

A pesar de las facilidades de las TIC para crear entornos virtuales que permitan el aprendizaje colaborativo, es el estudiantado quien de manera individual decide participar y trabajar en dichos ambientes. Podemos entender este aprendizaje como el proceso por el cual el individuo asimila, comprende e interioriza diferentes, conceptos, habilidades y competencias según sus preferencias a un ritmo y velocidad con la que se sienta seguro y sea capaz, posteriormente, de compartir, emplear, replicar, generar y demostrar la comprensión de la temática.

Según el Centre for Learning and Performance Technologies (2016), es: "Cualquier software o herramienta o servicio en línea que se puede utilizar para su propio aprendizaje personal o para la enseñanza o la formación". El aprendizaje individual puede ser ejemplificado como la asimilación de información convertida en experiencia por mecanismos como la repetición de tareas, el enfrentamiento y la resolución de problemas, la ejecución de proyectos, la comunicación y la interacción con diferentes elementos y la vivencia de situación en el transcurso del tiempo. Este proceso es fortalecido por mecanismos como las TIC que logran vincular los sentidos cognitivos con ambientes favorables para el aprendizaje personal.

"Por tanto, el modelo de aprendizaje individual está compuesto por un ciclo de aprendizaje conceptual y organizacional el cual es alimentado por los modelos mentales 
propios de la persona" (Caraballo, 2006, p.314). Otra postura hace mención al aprendizaje individual:

Está orientado a satisfacer necesidades del estudiante que pueden variar en el tiempo, la forma, el contenido y el volumen. Esto determina la necesidad de que los ambientes desarrollados para apoyar el Aprendizaje Individual sean flexibles, amigables y tengan incorporado los conceptos de adaptación. (Arteaga y Fabregat, 2002, p. 109)

Se desprende de las ideas anteriores que la asimilación y la creación del conocimiento es inherente a la persona, y para lograr los objetivos de aprendizaje es importante contar con ambientes capaces de adaptarse según las necesidades de tiempo, espacio, disponibilidad y accesibilidad e incorporar estos cambios con la misma flexibilidad con que aparecen los avances en tecnología de la comunicación. Del mismo modo, el profesorado debe incorporar nuevas competencias en su formación profesional de manera que pueda renovarse e innovar en su quehacer diario y busque ser un docente transformador.

\subsection{Innovación docente (docente transformador)}

Se entiende como el conjunto de acciones y esfuerzos realizados de manera planificada por medio de la experimentación e incorporación de cambios en el uso de nuevos instrumentos tecnológicos, que permitan la búsqueda de la autoevaluación y el mejoramiento continuo, respetando los objetivos de aprendizaje y empleando una correcta fundamentación teórica y didáctica.

Ibáñez (2004) la define como: "una respuesta a las necesidades y carencias que producen mejora, cambios que responden a un proceso planeado, deliberativo, sistematizado e intencional" (p.4). También expone la importancia de conjuntar los hechos, personas, situaciones e instituciones que intervienen en un momento dado para lograr un objetivo planteado.

La innovación docente se refiere al conjunto de acciones que generan cambios en busca de mejoras en los procesos de enseñanza-aprendizaje; estas acciones pueden tener su inicio como respuestas a diferentes necesidades o carencias de cada contexto educativo. El proceso de cambio debe ser planeado, administrado y sistemático con la intencionalidad de encontrar soluciones y mejoras que respondan a los diferentes actores: docente-estudiante, instituciones, o culturales. 
"Para responder a estos desafíos, las instituciones educativas deben revisar sus referentes actuales y promover experiencias innovadoras en los procesos de enseñanzaaprendizaje apoyándose en las TIC" (Ibáñez, 2004, p.2). De igual forma, este mismo autor señala la importancia de concentrar esfuerzos, no en la disponibilidad y potencialidad de las tecnologías; en su lugar se debe centralizar esfuerzos en los procesos de innovación docente: la docencia, estrategias didácticas, sistemas de comunicación y distribución de materiales de aprendizaje.

\section{Metodología}

\subsection{Sujetos participantes}

La investigación se realizó en el 2014 en el Recinto Universitario de Grecia de la Universidad de Costa Rica y tuvo una duración de un semestre. Participaron en la investigación un total de 15 estudiantes, con edades comprendidas entre 19 y 23 años, 2 de ellas mujeres y 13 hombres. Todos los estudiantes y las estudiantes pertenecían a la carrera de bachillerato en Informática Empresarial y estaban matriculados en el curso de Sistemas Operativos que se imparte durante el primer ciclo lectivo de marzo a julio, con una duración de 16 semanas, ubicado en el segundo año del plan de estudio.

\subsection{Método y objetivo}

La experiencia de innovación docente fue realizada con base en un enfoque cualitativo desde una aproximación descriptiva y reflexiva, que sistematiza el proceso, la experiencia y aprendizaje recolectado. El objetivo de la investigación fue innovar en la formulación de técnicas metodológicas y pedagógicas sobre ambientes colaborativos mediados por las TIC, que ayuden a involucrar al estudiantado-profesorado, como actores activos en los procesos de formación.

\subsection{Instrumentos de recolección de datos}

Se utilizaron varias herramientas para la recolección de datos durante las sesiones semanales de trabajo (un total de 32). Estas fueron registradas en la bitácora del investigador, únicamente las observaciones más destacadas de cada semana.

También se crearon dos formularios de Google Doc, uno registraba las preguntas que el estudiantado sugería al profesor después de terminar un tema de estudio. De igual forma, se registraba el día, la hora, el nombre del estudiante creador, el correo electrónico y se le 
colocaba un número consecutivo en el orden en que las preguntas fueron guardadas. Todos los estudiantes tenían acceso a esta información, por lo que, si una pregunta se repetía, era eliminada y se tenía que reformular otra pregunta en su lugar.

Por último, el profesor validaba cada una de estas preguntas y si era aceptada, el estudiante la guardaba en el segundo formulario, junto con una opción correcta y tres opciones falsas; pero si era rechazada, se le daba retroalimentación al estudiante para que cambiara la pregunta o la corrigiera para volver a subirla. También se utilizó un documento de Google Doc llamado Tabla de Fusión, que era alimentado con las preguntas válidas del segundo formulario, este documento es semejante a una hoja de cálculo. Fue utilizado como una base de datos para la aplicación Trivia, la cual se describe más adelante y su interfaz grafica se aprecia en el anexo 1. En ella, se guardaban las preguntas validadas y se registraba cada vez que un estudiante utilizada la aplicación.

Otra herramienta para recolectar datos fue la pizarra virtual, creada al inicio del semestre con el sitio web: padlet.com, la cual se puede observar en el anexo 2. Esta se utilizó para que el estudiantado pudiera dejar sus comentarios e impresiones sobre el ambiente virtual y el uso de la aplicación Trivia; todos los participantes tenían acceso a este recurso.

Por último, se empleó un instrumento de encuesta semiestructurada con preguntas abiertas y cerradas para registrar la opinión del estudiantado sobre el uso, la metodología y sobre sus impresiones de trabajar con este tipo de ambientes virtuales. El instrumento puede ser observado en el anexo 3. La encuesta fue aplicada en un único momento, una semana antes de la entrega de notas del curso y antes de finalizar el semestre, y fue completada por 10 estudiantes.

A continuación, se describe el proceso realizado durante cinco momentos, con suficiente claridad para responder al cómo y por qué se realizó el estudio.

\subsection{Primero momento: motivación}

El curso universitario IF4001 Sistemas Operativos se caracteriza por ser de tipo teóricopráctico; contiene una gran cantidad de conceptos complejos, que son indispensables para desarrollar los objetivos indicados en la propuesta programática. Estas cualidades generan una subutilización del método de clase magistral, el cual presenta debilidades como: la pérdida de interés del espectador, problemas para apropiar al estudiantado con sus aprendizajes; se enfoca en una parte del público académico, principalmente en aquellos estudiantes que se identifican con el aprendizaje por recepción. La participación activa y completa del 
estudiantado se ve limitada a unas cuantas aportaciones con preguntas dirigidas únicamente al profesorado.

La motivación por involucrar al estudiantado en la creación y utilización de contenidos teóricos provenientes de los libros de texto y de los materiales de estudio surgió desde las primeras etapas de planificación del curso. Por esta razón, se buscó una forma de vincular al estudiantado con él mismo y lograr un empoderamiento de las TIC, con el propósito de transformar y vincular el objeto de estudio (contenidos) con los sujetos involucrados (estudiantes y docentes).

Durante esta etapa, se identificó una aceptación de todos los participantes del curso con la utilización y el consumo de cualquier contenido proveniente de los dispositivos móviles (smartphone). Teniendo la motivación definida: vincular al estudiantado y al profesorado con los objetivos conceptuales del curso mediante las TIC con el propósito de que puedan apropiarse de sus aprendizajes, se da inicio al diseño y al modelado de una plataforma digital comenzando con una lluvia de ideas.

\subsection{Segundo momento: modelado}

En esta etapa se identificaron los siguientes elementos principales: las TIC, objetivos conceptuales y los participantes (docentes-estudiantes). Posteriormente, se comienza con la formulación de diferentes propuestas que logren entrelazar estos elementos de manera metodológica y mediante prácticas didácticas que fueran factibles de desarrollar y que fueran pertinentes al ambiente educativo e institucional de la sede. Es en este momento cuando surge la idea de utilizar la herramienta del cuestionario para complementar y ayudar a la aceptación y asimilación de los estudiantes con los objetivos conceptuales.

Las preguntas del cuestionario deberían ser todas de un mismo tipo de ítem para simplificar y reducir el tiempo de creación y facilitar el desarrollo del entorno mediado por las TIC. Así fue como se llegó a la conclusión de utilizar el ítem de selección única.

Como entorno de mediación, se escogió diseñar una aplicación móvil para el sistema operativo Android; ya que después de un sondeo durante la primera semana se descubrió que el cien por ciento del estudiantado contaba con acceso a dicho sistema operativo. Posteriormente, la aplicación requería estar disponible desde internet en todo momento, para satisfacer esta necesidad se emplearon los servicios de la nube computacional de Google AppsEngine; estos permitieron que cualquier usuario de dicha aplicación móvil pudiera tener acceso a las preguntas en cualquier momento y lugar, por medio de una conexión a internet; 
la cloud computing fue seleccionada entre otras disponibles en el mercado, debido a su disponibilidad, facilidad de uso, no tenía ningún costo económico y su excelente soporte técnico.

Se decide que el repositorio de preguntas sea confeccionado únicamente por los estudiantes; y que cada uno aporte un número pequeño de preguntas, entre una o dos, dependiendo del contenido textual utilizado en cada clase. Cada aporte debería ser realizado de manera voluntaria y posteriormente serían compartidas como material de aprendizaje colaborativo y además, responderían como complemento a las sesiones magistrales del curso.

Una vez delimitada la participación del estudiantado y definida la creación de los ítems, se busca una plataforma basada en las TIC que permita almacenar, compartir y poner en las manos de los participantes todas las preguntas creadas libremente sobre cada tema. La plataforma debería permitir la colaboración omnipresente entre los participantes, facilitando el acceso a todos los ítems de preguntas por medio de un dispositivo móvil. De esta manera, el estudiantado puede interactuar en el momento que quiera, según sus necesidades y preferencias personales, brindando facilidad y libertad de aprendizaje.

Las cualidades anteriormente descritas son suplidas de forma completa, simple y libre en la plataforma de Google AppsEngine, tal como se mencionó anteriormente. Entre los servicios utilizados se destacan: el correo electrónico, Google Docs y Google Drive. Mediante estas herramientas se crea un formulario compartido como enlace con los discentes para que suban los ítems creados. Posteriormente, la información del formulario es colocada de forma automática en otro documento de tipo tabla dinámica (tabla de fusión) de Google doc que tiene como función servir de base de datos o repositorio global con la información compartida.

Por último, se debía definir la manera de desarrollar una aplicación para dispositivos móviles que pudiera ser instalada en la mayoría de smartphone de las personas que integraban la clase. Además, la herramienta seleccionada tenía que ser simple de utilizar, intuitiva y con una curva de aprendizaje baja, para que docentes sin formación en programación pudieran usarla. Al realizar una indagación en la primera semana de clases para identificar la disponibilidad de dispositivos móviles y el tipo de sistema operativo empleaado por el estudiantado; se encontró que la totalidad de los participantes contaba con acceso a teléfonos inteligentes o tabletas con alguna versión del sistema operativo Android.

La información preliminar del sondeo indicó el camino para la escogencia de la plataforma de desarrollo de aplicaciones móviles para dispositivos Android llamada: appinventor, parte de la cual puede ser observada en el anexo 4. Esta herramienta tiene como 
misión la democratización del desarrollo de software por medio de la potenciación de todas las personas y convertirlas en creadoras de tecnología Massachusetts Institute of Technology (2012). Por medio de este entorno de desarrollo web fue posible la creación de la aplicación móvil (Trivia).

\subsection{Tercer momento: integración de conocimiento}

Partiendo de la base de crear una aplicación de Trivia alimentada y siendo consumida por el estudiantado como herramienta colaborativa, se inicia con la creación y el diseño del ambiente de aprendizaje colaborativo. Se crea un primer formulario (formulario de Google) que facilita la compartición de preguntas de tipo selección única; por simplicidad, facilidad y mantener un alcance real en la creación del ambiente. La creación de diferentes tipos de ítems de preguntas aumentaría exponencialmente el tiempo de desarrollo y no era parte del objetivo, por lo cual se decidió estandarizar las preguntas a un solo ítem de selección única, con la siguiente estructura: la primera casilla del formulario era para el texto de la pregunta, el siguiente para la respuesta correcta y, por último, tres campos para respuestas incorrectas, automáticamente se registraba también la hora, fecha y el nombre del creador de la pregunta.

Una vez utilizado este formulario, los ítems de preguntas son guardados en un documento de tipo hoja de cálculo de Google; posteriormente, por medio de un script se realiza la sincronización y el almacenado de forma automática de cada ítem al documento final de tipo tabla dinámica de Google. Dicho documento permite ser consultado vía internet igual que una base de datos remota; esta tabla dinámica era la encargada de brindar la información que es usada en la aplicación móvil (Trivia). La vinculación entre la tabla dinámica y la aplicación Trivia fue realizada por medio de la plataforma Google AppsEngine, la cual permite crear y configurar un servicio web para usuarios básicos que posean una cuenta de correo de gmail. El servicio permite realizar veinte cinco mil consultas diarias con un límite de dos solicitudes simultáneas por segundo por usuario; esta tecnología es la que permite enlazar un dispositivo móvil al banco de preguntas y brindar ubicuidad por medio de internet.

Después de esto se crea la aplicación móvil Trivia, la cual es elaborada en una primera versión únicamente con las características básicas: cargar preguntas, mostrar la pregunta de selección única y permitir la selección de una opción. Por ser la aplicación de carácter pedagógico, se incorpora la cualidad de no poder avanzar a la siguiente pregunta hasta no haber contestado correctamente. Esta primera versión definida como prototipo demostró que 
era posible crear un entorno virtual personalizado que funcionara como mediador entre el estudiantado y su aprendizaje, utilizando la cualidad de la colaboración.

El tiempo para la creación de la primera versión completamente funcional fue de ocho horas, repartidas en cuatro días de trabajo y se finalizó en la primera semana lectiva del curso. Es importante recalcar que todo el trabajo fue realizado únicamente por el profesor o profesora del curso. Los resultados obtenidos de la puesta en funcionamiento del entorno virtual se describen en su correspondiente apartado.

\subsection{Cuarto momento: ejecución y puesta en funcionamiento}

Luego de definida la metodología de trabajo y con la aplicación móvil funcionando, se entregó el archivo de instalación del prototipo de la aplicación Trivia a todos los participantes que quisieran utilizarlo como ayuda académica; todos los estudiantes del curso decidieron utilizarla. Al mismo tiempo, se compartió el acceso al formulario (Google Form) con el cual se pueden subir los ítems de preguntas y se explica el trabajo que debe realizar cada estudiante para crear y compartirlas. Básicamente, la persona estudiante agregaba una o dos preguntas de la materia vista en clases con una respuesta correcta y tres respuestas incorrectas. Las preguntas agregadas se guardaban en una hoja de cálculo de Google Doc. Estas tenían que ser únicas, no se podrían repetir. De esta forma, un estudiante diseñaba una pregunta, pero tenía acceso a 14 preguntas más (en un grupo de 15 participantes).

Luego de entregar la aplicación y establecer las pautas para el uso de esta; en las lecciones habituales del curso (se utilizó durante 14 semanas), se realiza la presentación habitual del trabajo de cada lección mediante clases magistrales donde se exponen las temáticas propias del curso de Sistemas Operativos y se invita al estudiantado a utilizar la herramienta Trivia luego de concluida cada clase. (En el anexo 1 encontrará imágenes de la interfaz de dicha aplicación). Además se creó una especie de pizarra virtual mediante el sitio web: http://es.padlet.com, el cual permitió al estudiantado escribir texto y colocar imágenes, link, entre otros.

El propósito era recolectar opiniones durante el uso de la aplicación. Los aportes obtenidos mediante Padlet evidencian las necesidades del estudiantado, carencias y solicitudes de cambios que fueron valorados para su incorporación según su importancia, pertinencia y su intencionalidad pedagógica. En el anexo 2 se puede observar una imagen de la pizarra virtual utilizada. 
Con la puesta en funcionamiento de la Trivia se creó un ambiente de interacción que involucró al estudiantado como parte de una experiencia creadora de recursos que fueron reintegrados para su propio aprendizaje. Se brindó la libertad de utilizar la aplicación móvil en el momento cuando se quisiera, fuera de las horas lectivas y desde distintos lugares. Este periodo de pruebas tuvo una duración de cuatro semanas con un total de ocho horas lectivas por semana. Pasado este momento, se generó una cantidad importante de datos que son expuestos en el siguiente momento y que se emplearon para mejorar el entorno virtual.

\subsection{Quinto momento: reconstrucción}

Después de un periodo de escrutinio y utilización de la aplicación Trivia, se analizan los primeros datos e impresiones logradas mediante la observación en clases y el seguimiento del uso de las consultas generadas por los estudiantes. Dichas consultas eran registradas por la plataforma Google AppsEngine al utilizar la aplicación por medio de internet y de las provenientes del cuarto momento. Se quiere destacar que este tipo de información se podría emplear para realizar un seguimiento sobre los tiempos de uso de este tipo de herramientas y el desempeño académico e intentar identificar patrones que puedan mejorar sus aprendizajes.

Junto a los datos anteriores se involucra al estudiantado por primera vez en el diseño y creación de una nueva versión. Se parte con la explicación del funcionamiento del entorno virtual colaborativo y se enseñan todos los aspectos técnicos como lo son: appinventor, Google AppsEngine y las diferentes herramientas de las TIC utilizadas.

Se replantean las observaciones del funcionamiento de la aplicación en colaboración con el estudiantado; aceptando o rechazando los posibles cambios al primer prototipo realizado por el docente y según la pertinencia de estos. A continuación, se mencionan los cambios más destacados durante este proceso:

- La sincronización automática entre el formulario utilizado para subir ítems y la tabla dinámica que brinda la información a la aplicación Trivia es eliminada, ya que se descubrió que algunas preguntas podían estar mal formuladas, tener errores o no estar acorde con los objetivos de aprendiza.

- Se incorpora un paso de verificación y aceptación de las preguntas antes de compartir un ítem; en este paso se acepta, niega o se corrigen las preguntas suministradas por el estudiantado. Se destaca que es el mismo estudiante quien realiza esta tarea, según las recomendaciones hechas por el docente.

- No se permite repetir preguntas sobre un mismo concepto. 
- Se identifica a la persona autora de cada ítem.

- La interfaz gráfica es mejorada y se agregan sonidos.

- Es incorporada una base de datos interna en la aplicación, permitiendo descargar el banco de preguntas y, posteriormente, poder utilizar la aplicación sin necesidad de tener conexión a internet permanente.

- Las preguntas son divididas por temas de estudio.

Este último momento es el comienzo nuevamente de la etapa llamada motivación, dando lugar a una metodología en forma de espiral. Tiene la intención de retomar la información de los momentos anteriores y aplicarla en el mejoramiento de los ambientes virtuales como medio de aprendizaje. También se permite la personalización de las experiencias de aprendizaje del estudiantado e impulsa la innovación docente guiada por un propósito educativo y pedagógico.

Al finalizar el semestre y antes de ser entregadas las calificaciones del curso, se aplicó al estudiantado un instrumento con preguntas semiestructuradas que permitieran recolectar las impresiones finales sobre la metodología empleada y la incorporación de la aplicación móvil, como parte del proceso de enseñanza-aprendizaje. El instrumento fue anteriormente validado por dos profesores de la carrera con experiencia en el curso y su diseño final se encuentra en el anexo 3.

\section{Resultados}

A continuación, se expone un modelo como propuesta, que puede ser utilizado para generar entornos de enseñanza-aprendizaje colaborativos mediados por las TIC. De igual forma, se presenta el ambiente y la arquitectura diseñada y desarrollada, producto de la investigación. Por último, se presentan las opiniones y experiencias del estudiantado, luego de haber trabajado con la aplicación Trivia y con el ambiente colaborativo, se recopilan antes de finalizar el semestre mediante la aplicación de un instrumento autoadministrado.

- Se obtiene como resultado una propuesta para un modelo de cinco momentos en espiral, que permite integrar los objetivos pedagógicos del profesorado y la experiencia del estudiantado, con el fin de interiorizar nuevas formas de emplear las TIC de manera integral, utilizando los conocimientos y recurso disponibles para cualquier miembro universitario: docente o estudiante, el cual se detalla en la figura 1. Este debería ser aplicado y evaluado de forma consensuada en otros 
estudios, para identificar su verdadero valor como guía en la creación de recurso pedagógicos innovadores.

Figura 1: Modelo de 5 momentos.

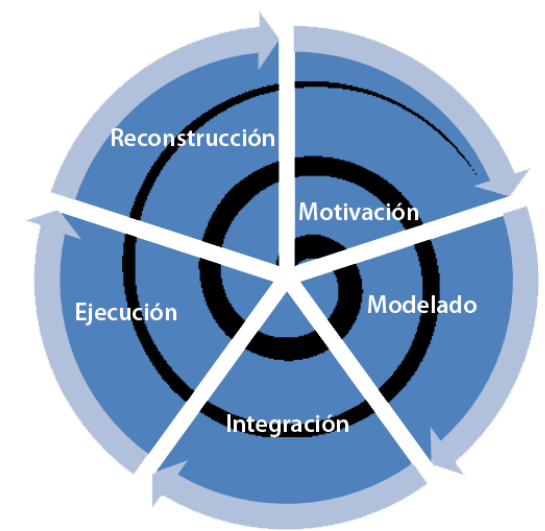

Fuente: Elaboración propia a partir de la sistematización de las experiencias y aprendizajes recolectados

- Se logró la creación de un entorno de aprendizaje virtual patrocinado con elementos tecnológicos accesibles de forma libre y al alcance de cualquier docente o estudiante universitario con acceso a internet. Se detalla en la figura 2, el entorno virtual resultante y, además, en la figura 3, se describe la implementación técnica utilizada. A pesar de las facilidades evidenciadas sobre el uso de las TIC para la creación de dicho entorno, se invita a otros investigadores a cuantificar las verdaderas cualidades y habilidades docentes requeridas para trabajar con este tipo de tecnología.

Figura 2: Entorno virtual colaborativo.

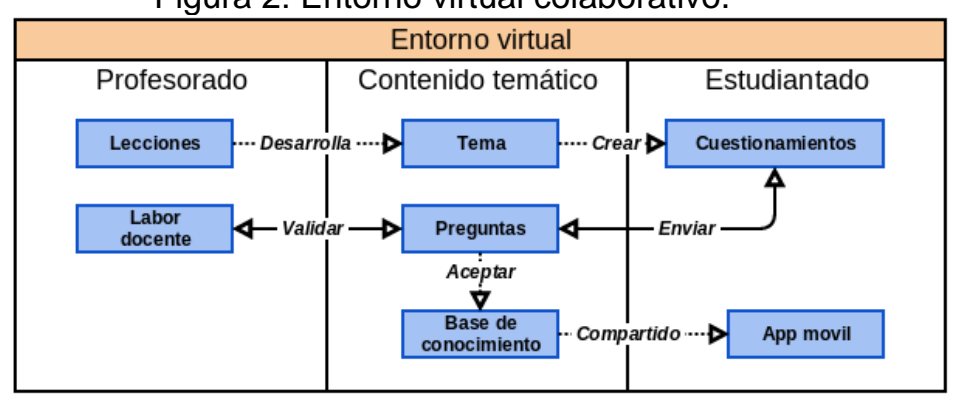

Fuente: Elaboración propia a partir de los datos obtenidos durante la utilización de la aplicación móvil. 
Figura 3: Modelo de la arquitectura desarrollado.

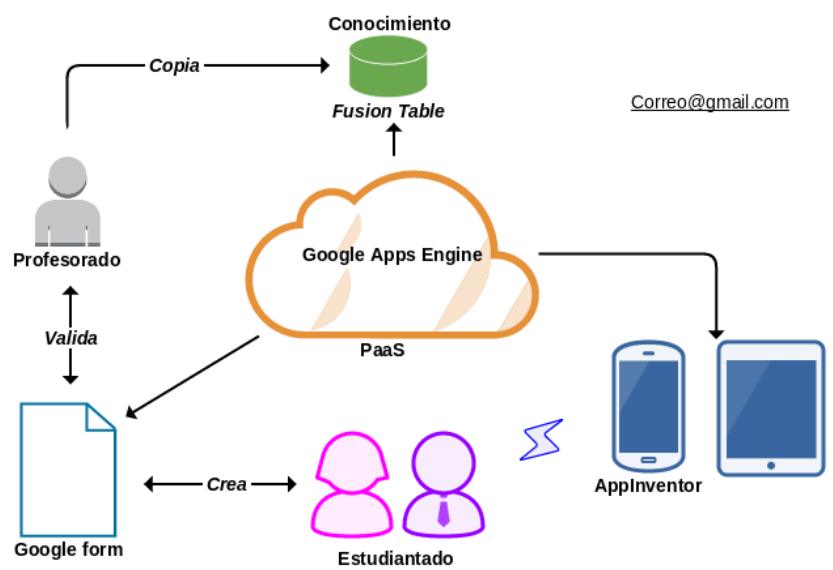

Fuente: Elaboración propia a partir de la implementación técnica realizada.

- La metodología anteriormente descrita puede ser empleada de forma satisfactoria por distintos docentes de diversas áreas del saber, con conocimientos y habilidades mínimas en el manejo de las TIC. Puede ser aplicada para acercar a grupos de clases y a estudiantes individuales mediante herramientas TIC, definiendo y delimitando su área del saber correspondiente.

- Se comprobó que existe tecnología que está al alcance para ser modelada según las necesidades académicas; $Y$, al mismo tiempo, poder interiorizarla en función del proceso de enseñanza-aprendizaje de cada docente-estudiante. Este recurso no debe ser visto como un bien o un servicio que simplemente es consumido y que no es posible transformarlo o es inamovible.

- En este tipo de procesos de mutua colaboración es indispensable incorporar al docente como un mediador, es el encargado de esclarecer, verificar y sugerir las correspondientes mejoras o cambios a cada estudiante sobre sus aportes. Con la intención de poder lograr la creación de contenidos de aprendizaje de calidad, para mantener la motivación y la correcta participación del estudiantado.

- Se logró involucrar de manera activa al estudiantado en espacios tanto internos como externos a las lecciones presenciales por medio de los entornos virtuales; los cuales permitieron la realización de actividades en un ambiente colaborativo y pedagógico durante las clases, incentivando la participación de los participantes. 
- El entorno virtual permitió la interacción de los elementos: estudiantado, profesorado, aplicaciones móviles y los contenidos temáticos, guiados por una intencionalidad pedagógica. Las interacciones entre ellos fueron formales durante el tiempo de las lecciones, pero, fuera de las horas del curso, estas se realizaron de manera informal y asincrónica, cada participante interactuaba en el instante que consideró pertinente.

A continuación, se presentan los resultados de la aplicación del instrumento. Este fue completado por un total de 10 estudiantes de un grupo de 15, con edades comprendidas entre 19 y 23 años, 2 de ellas eran mujeres y 8 eran hombres. Los participantes indican, con respecto a la utilización de dispositivos móviles como medio de aprendizaje, lo siguiente:

- Al consultar sobre sí el uso de aplicaciones móviles fue de utilidad para su aprendizaje, el $100 \%$ de las respuestas emitidas fueron positivas, algunas razones escritas son: "se aprende jugando", "entretenido y con interacción con los estudiantes", "me permitió estar practicando la materia de cada capítulo después de estudiarlo y así saber que tanto había aprendido", "el curso tiene mucha materia teórica y es más fácil de esta manera lograr aprender", "ayuda para repasar y ver en qué temas hay que reforzar el estudio".

- Al pedir la opinión sobre el uso de cuestionarios colaborativos mediados con aplicaciones móviles, el $100 \%$ de las respuestas evidencian la aceptación por parte de los participantes y algunas descripciones destacadas son: "se aprende, divierte y entretiene a la vez", "es muy útil porque nosotros somos los que nos preguntamos, lo más importante de X tema", "es un modo de hacer un repaso rápido de la materia".

- En relación con la pregunta sobre si le gustaría que se continúe utilizando esta metodología mediada por aplicaciones móviles, el 100 \% respondió que sí; algunas respuestas fueron: "son fáciles de utilizar, agilizan el aprendizaje de una forma más interactiva", "podía aprovechar el tiempo cuando no estaba en la universidad o el apartamento", "se hace más fácil estudiar", "gracias a ella podía saber de qué cosas me acordaba menos y poder repasarlas".

- Ninguno de los 10 participantes que completaron el instrumento había tenido anteriormente experiencias con aplicaciones móviles como medio de aprendizaje, en otros cursos, universidades, ni en centros académicos. 


\section{Conclusiones}

El estudio nos invita a trabajar varios aspectos. Por un lado, el profesorado debe asumir un rol orientador y mediador; debe crear situaciones dentro del aula y fuera de esta por medio de las TIC. Al mismo tiempo, debe guiar la interacción entre los estudiantes y diseñar herramientas que faciliten el proceso enseñanza-aprendizaje, mediante elementos de la tecnología y supervisar su correcta aplicación en función de los objetivos de aprendizaje.

Por otro lado, el estudiantado está en obligación de asumir un papel más protagónico e involucrarse en los procesos de su enseñanza-aprendizaje. Asimismo, debe aportar su conocimiento sobre las TIC y apropiarse de estas, para mejorar sus experiencias educativas. Estar abiertos y dispuestos a la colaboración con sus compañeros y tomar un papel más activo, dentro y fuera del espacio académico de la mano con el profesorado.

Se concluye que un ambiente colaborativo virtual facilita la colaboración entre estudiante-estudiante y docente-estudiante, permitiendo al estudiantado tomar sus decisiones mediante la creación de sus propios recursos de aprendizaje; ellos identificaron conceptos e ideas según la importancia para sí mismos y para sus compañeros. Este tipo de espacios logra involucrar al estudiantado en el uso y el diseño de herramientas que ayuden en sus procesos educativos.

El estudiantado cuenta con competencias en las nuevas tecnologías, pueden utilizarlas con naturalidad, facilidad y propiedad. Sin embargo, se evidencia una deficiencia en el momento de crear, modificar o de apoderarse de las TIC y dejar de ser simples usuarios. No son capaces de apropiarse de la aplicación y moldearla según sus necesidades, inquietudes y gustos individuales.

Existe una brecha digital entre el uso para el aprendizaje y llegar a ser dueños de sus aprendizajes, mediante la tecnología; en ser creadores e innovadores de tecnología. Para el estudiantado fue difícil redactar las preguntas y valorar cuáles ideas eran las de mayor importancia según los objetivos del curso; querían redactar preguntas sobre todo y de cualquier tema sin importar los objetivos.

Por lo indicado anteriormente, fue necesario que el profesorado filtrara y supervisara cada uno de los ítems de preguntas; esto se solucionó con la creación de un segundo formulario para proponer preguntas, del cual el profesorado tomaba las más importantes según su experticia para compartirlas en la Trivia y al mismo tiempo le indicaba las correcciones o sugerencias al autor de cada una de ellas para que fueran mejoradas. Para ellos era más difícil enfrentarse a la redacción de una pregunta, que buscar una respuesta. 
Los resultados del instrumento indican que 9 participantes respondieron todas las preguntas disponibles de la aplicación antes de realizar los exámenes del curso; evidenciando que la utilización y los aprendizajes son individuales, ya que es el estudiantado el responsable de invertir parte de su tiempo, fuera del ambiente presencial universitario; tiempo que es invertido en el uso del entorno virtual y en el sostén del ambiente mediante las aportaciones personales que fueron realizadas por todos los participantes.

Los entornos virtuales individuales representan una forma de autoevaluación y retroalimentación de forma inmediata y pertinente, ayudando al estudiantado a replantear su aprendizaje actual. El ambiente de aprendizaje se convirtió en una primera fuente de autoevaluación que permitió al estudiantado contar con una pequeña, pero importante estimación de lo estudiado en un momento dado y sobre un tema en particular. Estas primeras indicaciones brindaron cierto nivel de seguridad frente a las evaluaciones escritas. Es importante indicar que el entorno virtual propuesto es simplemente una unión, un vínculo para entrelazar: estudiantes, docentes y contenidos como una opción de ayuda y mejoramiento del proceso de enseñanza-aprendizaje; principalmente en temáticas con alto contenido teórico.

Se invita al lector a reformular este tipo de trabajo como un vínculo hacia otras áreas del saber universitario, con la intención de continuar su validación y reformular ideas e incorporar mejoras al proceso aquí planteado; Al mismo tiempo, se exhorta a expandir la utilización de herramientas TIC en las prácticas pedagógicas en función de sus beneficios de inmediatez y accesibilidad.

En este primer acercamiento, se introdujeron importantes hallazgos en materia de trabajo colaborativo, prácticas pedagógicas docentes, uso y creación de las TIC personalizadas a necesidades académicas específicas. Es pertinente continuar con el perfeccionamiento de la presente metodología mediante la aplicación de las correcciones recolectadas al final de dicha sistematización. Este estudio continúa abierto con el propósito de enriquecerlo mediante la incorporación de futuras entregas con resultados provenientes de otras técnicas; como por ejemplo: grupos focales, proyecto de casos entre otros.

\section{Referencias}

Alcina Caudet, Amparo. (2002). Tutorizar trabajos terminológicos en un entorno virtual de trabajo colaborativo. Sendebar, (13), 169-181.

Arteaga, Carlos y Fabregat, Ramón. (2002). Integración del aprendizaje individual y del colaborativo en un sistema hipermedia adaptativo. JENUI, 2(2), 107-114. 
Ascheri, María Eva, Testa, Oscar, Pizarro, Rubén, Camiletti, Pablo y Diaz, Lucas. (2014). Desarrollo de aplicaciones para dispositivos móviles con sistema operativo Android para la enseñanza aprendizaje de temas de matemáticas en el nivel medio. En XVI Workshop de Investigadores en Ciencias de la Computación. Recuperado de http://sedici.unlp.edu.ar/bitstream/handle/10915/43878/Documento completo.pdf?sequ ence $=1$

Bertolín, Javier Areitio. (2010). Protección del Cloud Computing en seguridad y privacidad. Revista española de electrónica, (666), 42-48.

Bianchi Ferreyra, Eugenia, Lombardero, Oscar Guillermo, Fernández, Mirta y Barrios, Walter. (2014). Mobile y Cloud: plataforma de desarrollo Android, experiencia de aprendizaje en robótica. En XX Congreso Argentino de Ciencias de la Computación (Buenos Aires, Argentina). Recuperado de http://sedici.unlp.edu.ar/handle/10915/42281

Calzadilla, María Eugenia. (2002). Aprendizaje colaborativo y tecnologías de la información y la comunicación. Revista Iberoamericana de educación, 1-10.

Caraballo, Martínez, Noemí. (2006). Gestión del Conocimiento: Aprendizaje individual versus aprendizaje organizativo. Intangible Capital, 2(13), 308-326.

Carrió Pastor, María Luisa. (2007). Ventajas del uso de la tecnología en el aprendizaje colaborativo. Revista Iberoamericana de Educación, 41(4),1-10.

Catasús Montse, Guitert, Romeu, Teresa y Pérez-Mateo, María. (2007). Competencias TIC y trabajo en equipo en entornos virtuales. Revista de Universidad y Sociedad del Conocimiento, (4), 1-12.

Centre for Learning and Performance Technologies. (2016). Top Tools for Learning 2016. Centre for Learning and Performance Technologies. Recuperado de http://c4lpt.co.uk/top100tools/

Cobo Romaní, Juan Cristóbal. (2011). El concepto de tecnologías de la información. Benchmarking sobre las definiciones de las TIC en la sociedad del conocimiento. ZerRevista de Estudios de Comunicación, 14(27), 295-318 Recuperado de http://www.ehu.eus/zer/hemeroteca/pdfs/zer27-14-cobo.pdf

Conde González, Miguel Ángel. (2007). mLearning, de camino hacia el uLearning. Recuperado de http://gredos.usal.es/jspui/bitstream/10366/21829/1/TM mLearningcamino.pdf

Esteve, Francesc. (2009). Bolonia y las TIC: de la docencia 1.0 al aprendizaje 2.0. La cuestión universitaria, 5, 59-68.

Ferro Soto, Carlos, Martínez Senra, Ana Isabel, y Otero Neira, María del Carmen. (2009). Ventajas del uso de las TICs en el proceso de enseñanza-aprendizaje desde la óptica de los docentes universitarios españoles. Edutec-e: Revista electrónica de tecnología educativa, 29 (2009), 1-12. Recuperado de file://C:/Users/ACASTI 1/AppData/Local/Temp/788871931.ART 1 Ventajas\%20TICS1.pdf 
Ibáñez Salinas, Jesús. (2004). Innovación docente y uso de las TIC en la enseñanza universitaria. Revista Universidad y Sociedad del Conocimiento, 1(1). Recuperado de http://dialnet.unirioja.es/descarga/articulo/1037290.pdf

Imperatore, Adriana. (2009). Comunicación y educación en entornos virtuales de aprendizaje: perspectivas teórico-metodológicas. Argentina: Universidad Nacional de Quilmes Editorial.

Marino Aparici, Roberto. (2013). Conectados en el ciberespacio. Madrid, España: Editorial UNED

Massachusetts Institute of Technology. (2012-2015). What is MIT App Inventor?. Recuperado de http://appinventor.mit.edu/explore/about-us.html

Moreno Cely, Claudia y Molano Avendaño, Luz. (2012). El docente transformador de la cultura en la sociedad del conocimiento. XXVII Congreso Nacional y I Internacional de Lingüística, Literatura y Semiótica. Recuperado de http://www.uptc.edu.co/export/sites/default/eventos/2012/cnills/documentos/el docente transformador cultura.pdf

Observatorio de la formación en Red SCOPEO. (2011). M-Learning en España, Portugal y América Latina. Salamanca, España: Universidad de Salamanca Servicio de Innovación y Producción Digital. Recuperado de http://scopeo.usal.es/wpcontent/uploads/2013/04/scopeom003.pdf

Salinas, Jesús. (2005). Nuevos escenarios de aprendizaje. Grupo CIFO: IV Congreso de Formación para el Trabajo. Recuperado de http://www.researchgate.net/profile/Jesus Salinas/publication/232242510 Nuevos esc enarios de aprendizaje/links/02bfe5100ea5a7b332000000.pdf 


\section{Anexos}

En este apartado se incluyen imágenes de la aplicación móvil creada, de las herramientas de las TIC utilizadas y del instrumento empleado para recopilar los datos de las personas participantes en la investigación.

Anexo 1: interfaz principal de la aplicación Trivia

En este anexo se exponen imágenes de la interfaz del menú de la aplicación móvil (Trivia); en ella se aprecian las cuatro acciones principales: carga desde internet la base de datos de las preguntas, seleccionar un tema específico de preguntas, ir al juego de preguntas y, finalmente, mostrar los nombres de los estudiantes que colaboraron en el proyecto. También se puede observar la interfaz de la mecánica del juego con una pregunta y cuatro opciones de respuestas.

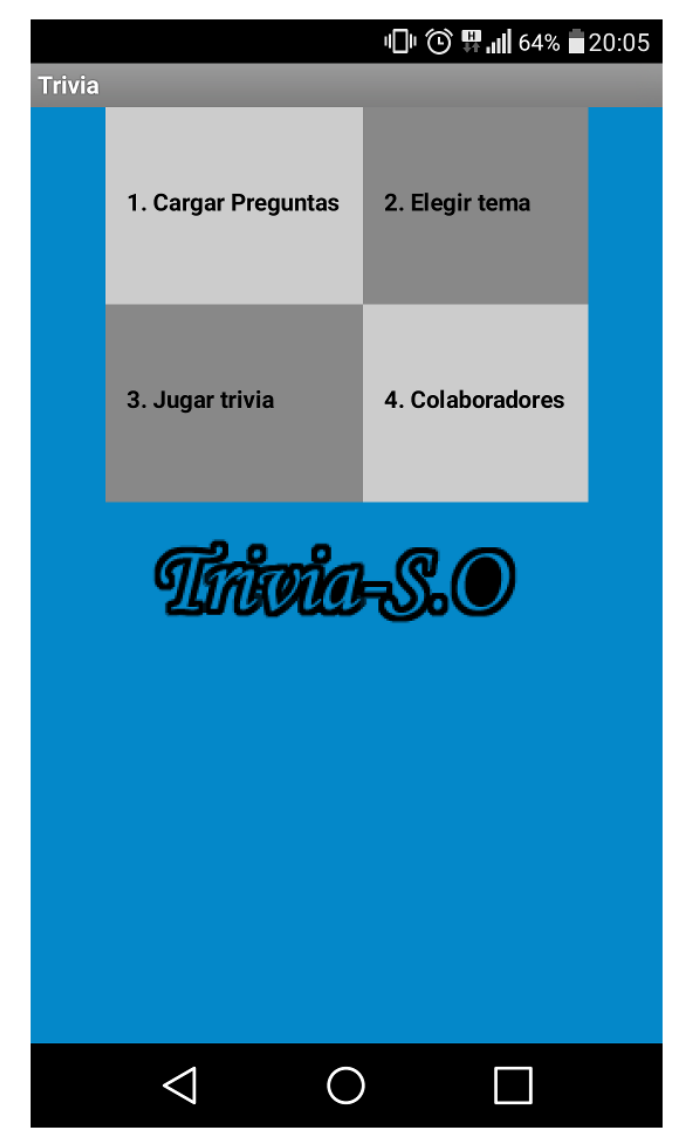

a) menu de la aplicación

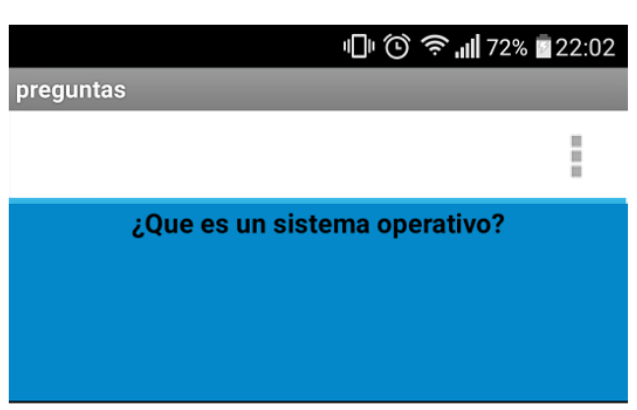

Es un SW intermedio entre el HW y los usuarios

Es un programa que le permite al usuario controlar las computadoras

Es el único SW encargado de administrar el hardware y realizar la comunicación con el resto del sistema de cómputo.

Es un SW intermediario entre los componentes físicos de un sistema de cómputo HW y el usuario, administrando de forma intuitiva y eficiente todos los recursos.

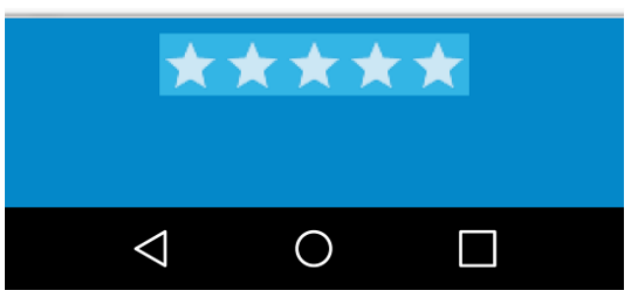

b) preguntas de la trivia 
Anexo 2: interfaz web de la herramienta Padlet, utilizada como pizarra virtual colaborativa, para la recolección de opiniones y comentarios sobre el prototipo de la trivia.

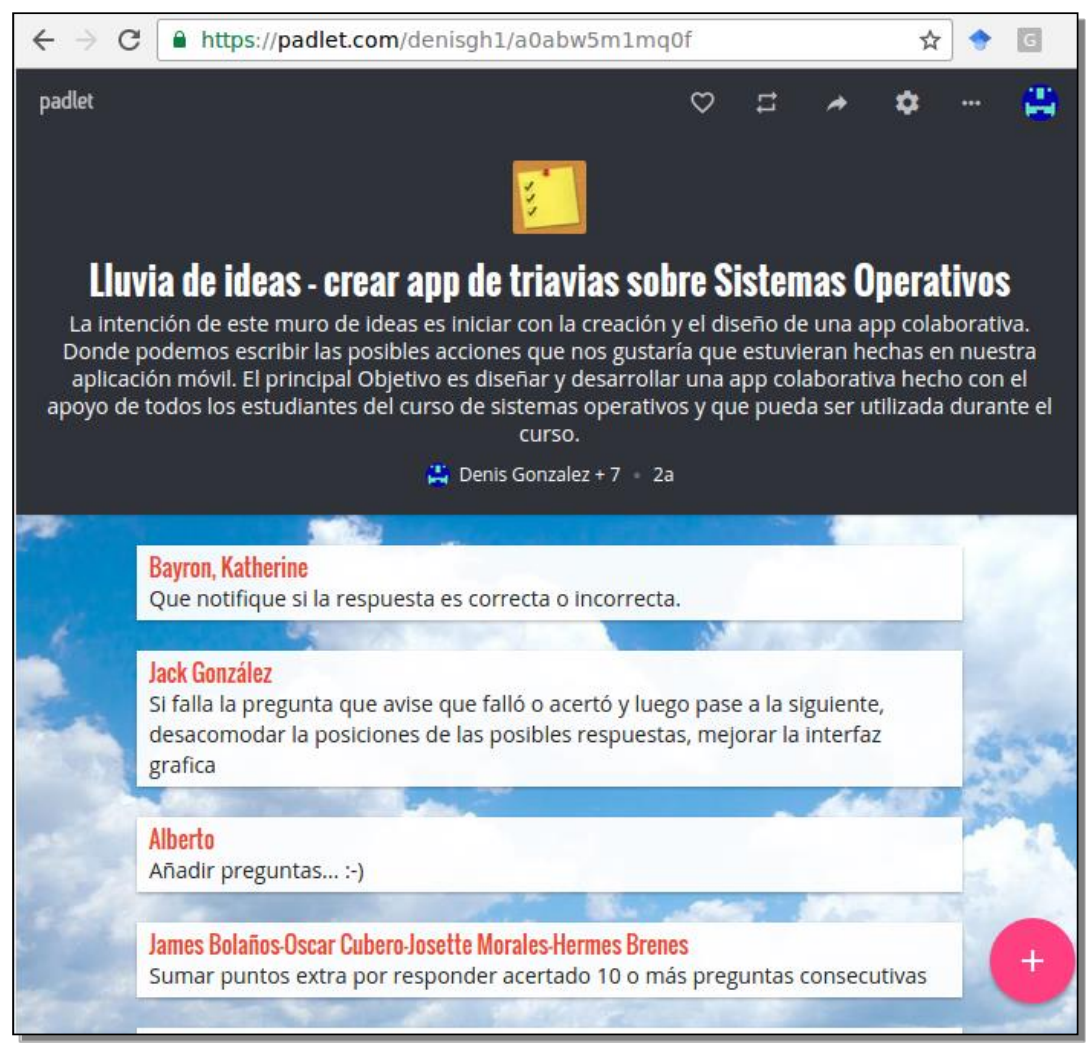




\section{Anexo 3.}

\section{Instrumento de evaluación del uso de la aplicación Trivia durante el curso de Sistemas Operativos}

Muchas gracias por participar. A continuación se presentan una serie de preguntas que pretenden registrar sus opiniones sobre el uso de la aplicación Trivia. No hay respuestas correctas o incorrectas todas sus ideas son de gran importancia.

1. Por favor indique su edad y sexo

2. ¿Cuál es su opinión sobre el uso de la aplicación móvil (Trivia)?

3. ¿Cuál es su opinión sobre el uso de cuestionarios colaborativos mediante aplicaciones móviles?

4. ¿ ¿Instaló y utilizó la aplicación (sí / no)? ¿Por qué?

5. ¿Le gustó el uso de la aplicación (Trivia-so) durante el curso? (sí / no) ¿Por qué?

6. ¿Aproximadamente cuántas preguntas respondió?

7. ¿Le gustaría que se continúe utilizando este tipo de aplicaciones en otros curso? (sí / no) ¿Por qué?

8. ¿Considera que aprendió mejor el contenido del curso con la ayuda de la aplicación móvil? (sí / no) ¿Por qué?

9. ¿Ha utilizado este tipo de aplicaciones en otros cursos académicos como recurso didáctico?

10. De todas las actividades desarrolladas durante el curso, cuál fue la más importante para su aprendizaje y por que.

Muchas gracias por su participación. 
Anexo 4: Plataforma de desarrollo de Appinventor, utilizada para la creación de la aplicación móvil Trivia.

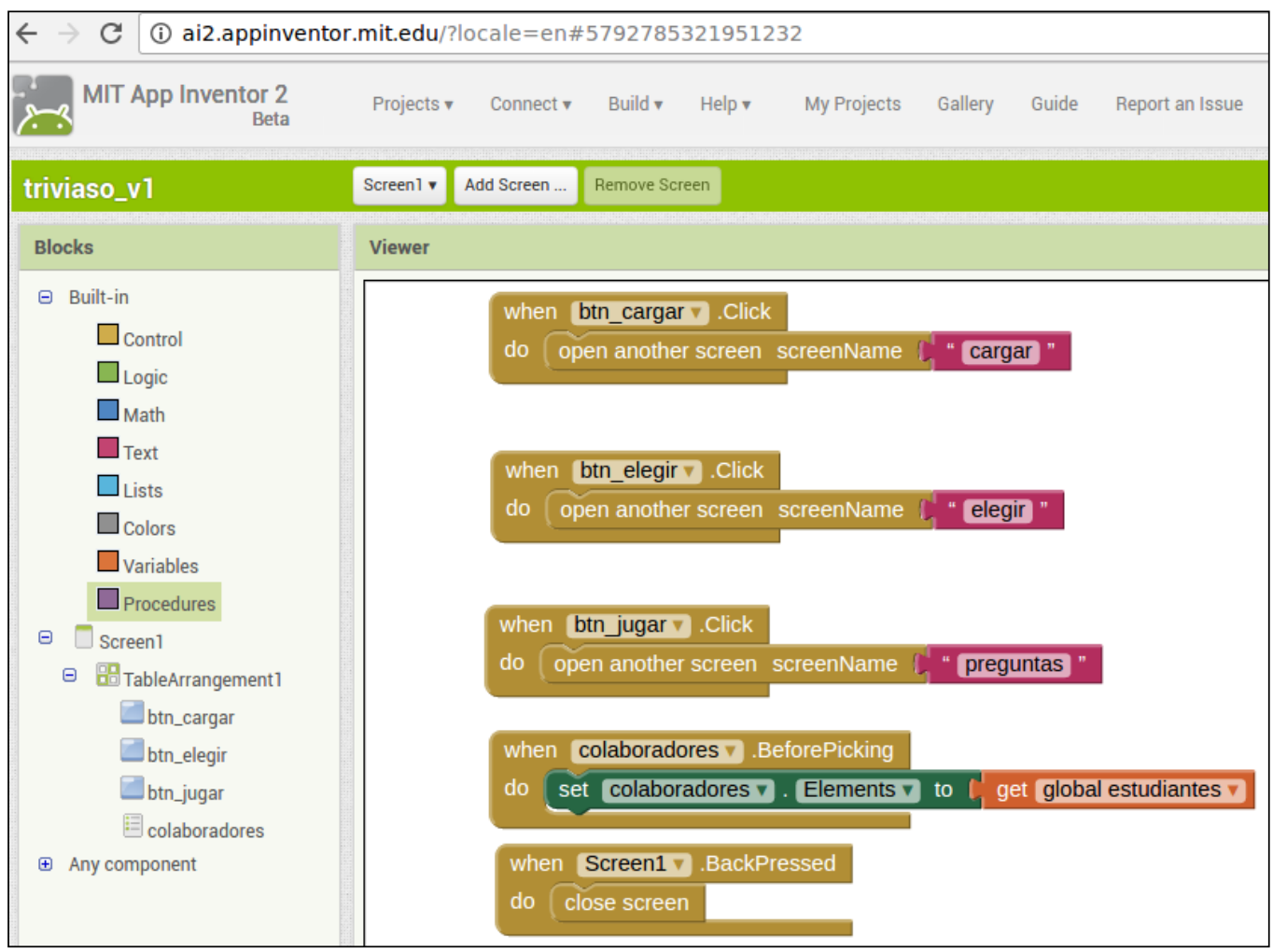

